\title{
A simple model to interpret the ultraviolet, optical and infrared SEDs of galaxies
}

\author{
Elisabete da Cunha ${ }^{1,2}$, Stéphane Charlot $^{3,4}$ \& David Elbaz ${ }^{5}$ \\ ${ }^{1}$ Department of Physics, University of Crete, 71003 Heraklion, Greece \\ ${ }^{2}$ IESL/Foundation for Research and Technology-Hellas, 71110 Heraklion, Greece \\ email: dacunha@physics.uoc.gr \\ ${ }^{3}$ UPMC Univ. Paris 6, UMR7095, Institut d'Astrophysique de Paris, 75014 Paris, France \\ ${ }^{4}$ CNRS, UMR7095, Institut d'Astrophysique de Paris, 75014 Paris, France \\ ${ }^{5}$ Laboratoire AIM, CEA/DSM-CNRS-Université Paris Diderot, IRFU/Service \\ d'Astrophysique, CEA-Saclay, 91191 Gif-sur-Yvette Cedex, France
}

\begin{abstract}
We present a simple, largely empirical but physically motivated model, which is designed to interpret consistently multi-wavelength observations from large samples of galaxies in terms of physical parameters, such as star formation rate, stellar mass and dust content. Our model is both simple and versatile enough to allow the derivation of statistical constraints on the star formation histories and dust contents of large samples of galaxies using a wide range of ultraviolet, optical and infrared observations. We illustrate this by deriving medianlikelihood estimates of a set of physical parameters describing the stellar and dust contents of local star-forming galaxies from the Spitzer Infrared Nearby Galaxy Sample (SINGS) and from a newly-matched sample of SDSS galaxies observed with GALEX, 2MASS, and IRAS. The model reproduces well the observed spectral energy distributions of these galaxies across the entire wavelength range from the far-ultraviolet to the far-infrared. We find important correlations between the physical parameters of galaxies which are useful to investigate the star formation activity and dust properties of galaxies. Our model can be straightforwardly applied to interpret observed ultraviolet-to-infrared spectral energy distributions (SEDs) from any galaxy sample.
\end{abstract}

Keywords. dust, extinction; galaxies: stellar content; galaxies: ISM; galaxies: statistics.

\section{Introduction}

Combined ultraviolet, optical and infrared data are now becoming available for large samples of galaxies. To extract constraints on the stellar populations and ISM of galaxies from these multi-wavelength observations requires the consistent modelling of the emission by stars, gas and dust. A standard approach to this problem is to compute the radiative transfer of the light emitted by evolving stellar populations through gas and dust in the ISM (e.g. Silva et al. 1998, Dopita et al. 2005). Such sophisticated models provide valuable insight into the detailed emission of individual galaxies. However, because of the complexity of radiative transfer calculations, these models are not optimised to derive statistical constraints on physical parameters from observations of large samples of galaxies. We present a simple, largely empirical but physically motivated model which can be used to interpret the mid- and far-infrared SEDs of galaxies consistently with the emission at ultraviolet, optical and near-infrared wavelengths. This model allows us to derive statistical estimates on physical parameters related to the star formation history and dust content of large samples of galaxies. 


\section{Description of the model}

We compute the emission by stars in galaxies using the latest version of the Bruzual \& Charlot (2003) population synthesis code (Charlot \& Bruzual, in prep.). The stellar emission is attenuated using the simple two-component dust model of Charlot \& Fall (2000), which accounts for the fact that stars are born in dense molecular clouds with typical lifetimes of $10^{7} \mathrm{yr}$; at later ages, stars migrate to the ambient (diffuse) ISM (see equation 2 in da Cunha et al. 2008). We use an 'effective absorption' curve for each component, $\hat{\tau}_{\lambda} \propto \lambda^{-n}$, where the slope $n$ reflects both the optical properties and the spatial distribution of the dust. We adopt $n=0.7$ for the ambient ISM and $n=1.3$ for the stellar birth clouds. This prescription allows us to compute the total energy absorbed by dust in the birth clouds and in the ambient ISM; this energy is re-radiated by dust at infrared wavelengths.

We define the total dust luminosity re-radiated by dust in the birth clouds and in the ambient ISM as $L_{\mathrm{d}}^{\mathrm{BC}}$ and $L_{\mathrm{d}}^{\mathrm{ISM}}$, respectively. The total luminosity emitted by dust in the galaxy is then $L_{\mathrm{d}}^{\text {tot }}=L_{\mathrm{d}}^{\mathrm{BC}}+L_{\mathrm{d}}^{\mathrm{ISM}}$.

We distribute $L_{\mathrm{d}}^{\mathrm{BC}}$ and $L_{\mathrm{d}}^{\mathrm{ISM}}$ in wavelength over the range from 3 to $1000 \mu \mathrm{m}$ using four main components:

- the emission from polycyclic aromatic hydrocarbons (PAHs; i.e. mid-infrared emission features),

- the mid-infrared continuum emission from hot dust with temperatures in the range $130-250 \mathrm{~K}$,

- the emission from warm dust in thermal equilibrium with adjustable temperature in the range $30-60 \mathrm{~K}$,

- the emission from cold dust in thermal equilibrium with adjustable temperature in the range $15-25 \mathrm{~K}$.

In stellar birth clouds, the relative contributions to $L_{\mathrm{d}}^{\mathrm{BC}}$ by PAHs, the hot mid-infrared continuum and warm dust are kept as adjustable parameters. These clouds are assumed not to contain any cold dust. In the ambient ISM, the contribution to $L_{\mathrm{d}}^{\text {ISM }}$ by cold dust is kept as an adjustable parameter. The relative proportions of the other 3 components are fixed to the values reproducing the mid-infrared cirrus emission of the Milky Way. We find that this minimum number of components is required to account for the infrared SEDs of galaxies in a wide range of star formation histories (see da Cunha et al. 2008 for details).

\section{The star formation activity and dust content of local galaxies}

The model summarised in the previous section allows us to derive statistical constraints on the physical properties of galaxies from ultraviolet, optical and infrared observations. We build a comprehensive library of stochastic models covering a wide range of star formation histories, metallicities, dust attenuation, dust temperatures and fractional contributions by different dust components to the total infrared luminosity. For each model in the library, we compute the predicted ultraviolet, optical and infrared fluxes in the GALEX, SDSS, 2MASS, Spitzer, IRAS, ISO and SCUBA bands. We compare these predictions with observations of two local samples of galaxies: the Spitzer Infrared Nearby Galaxy Sample (SINGS, Kennicutt et al. 2003), and a sample of 3258 SDSS galaxies with complementary photometric observations by GALEX, 2MASS and IRAS (da Cunha et al. 2009).

For each observed galaxy, we assess how each model in the stochastic library (characterised by a set of randomly drawn physical parameters) fits the observed SED by 


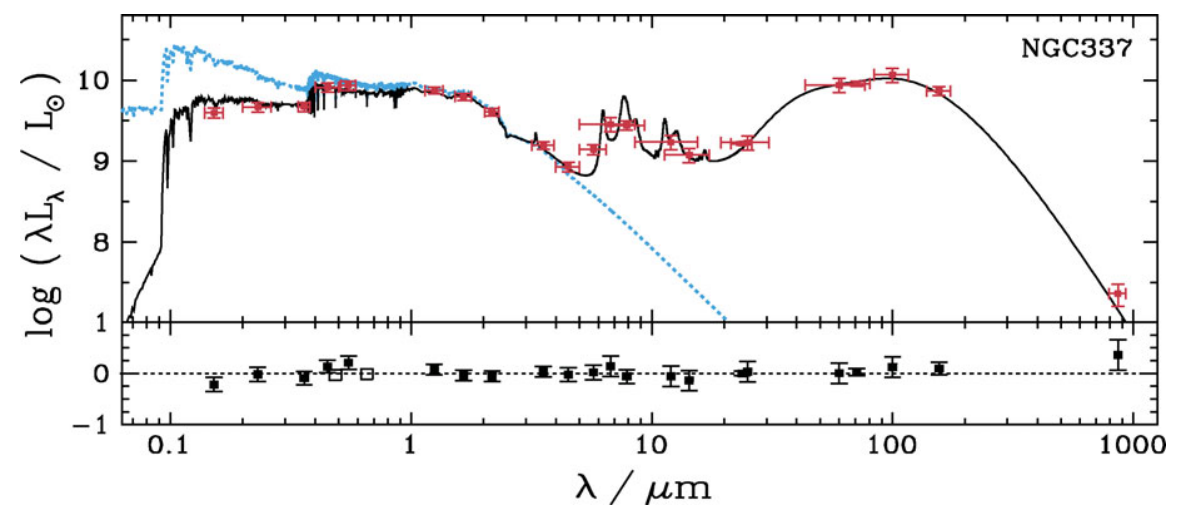

Figure 1. Best-model fit to the observed SED (red squares) of the SINGS galaxy NGC 337. The dashed-blue line shows the unattenuated stellar spectrum and the black line shows the total SED (i.e. stellar emission attenuated by dust + infrared dust emission). In the bottom panel, we plot the fit residuals, $\left(L_{\mathrm{obs}}-L_{\mathrm{mod}}\right) / L_{\mathrm{obs}}$, where $L_{\mathrm{obs}}$ is the observed luminosity and $L_{\mathrm{mod}}$.
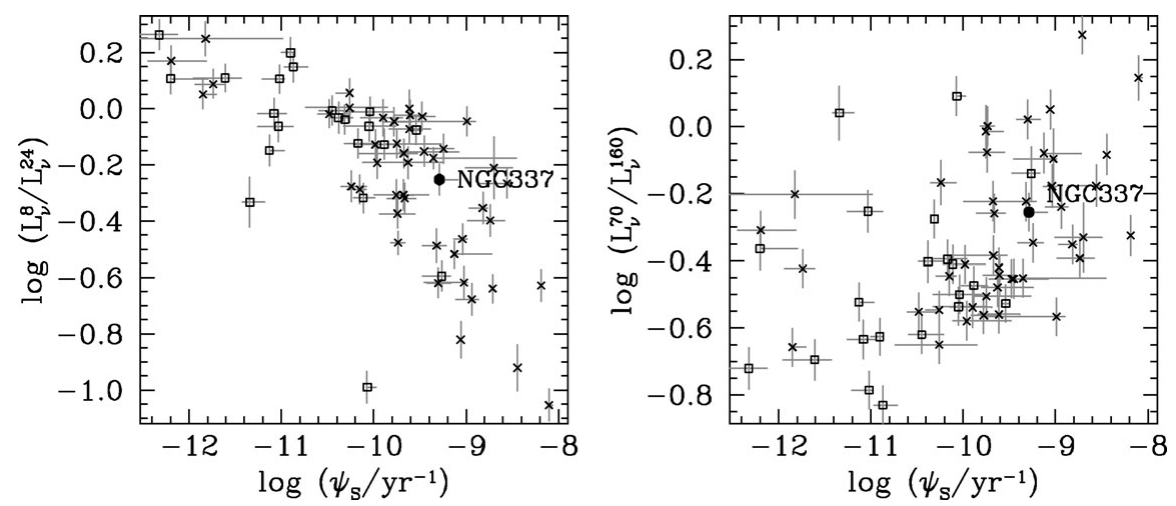

Figure 2. Spitzer infrared colours (left-ratio of 8- to $24-\mu \mathrm{m}$ luminosity; right-ratio of 70 - to $160-\mu \mathrm{m}$ luminosity) plotted against the median-likelihood estimate of the specific star formation rate, $\psi_{\mathrm{S}}$, derived for the galaxies in the SINGS sample.

computing the $\chi^{2}$ goodness-of-fit. We build the likelihood distribution of any physical parameter by weighting the value of that parameter in each model of the library by the probability $\exp \left(-\chi^{2} / 2\right)$. Our final estimate of the parameter is the median of the likelihood distribution and the associated confidence interval is the 16 th- 84 th percentile range.

\subsection{SINGS sample}

We start by exploiting our model to interpret observed SEDs of the SINGS sample, which contains 75 local galaxies spanning a wide range in morphology and star formation activity. We compare our model predictions with the ultraviolet, optical and infrared observations of these galaxies using the method outlined above. To illustrate the quality of our fits, in Fig. 1, we show an example of the best-fit SED of one galaxy of this sample with median Spitzer infrared colours, NGC 337.

The results of our spectral fits for the full sample show that the mid- and far-infrared colours of galaxies correlate strongly with the specific star formation rate (i.e. star formation rate divided by the stellar mass; see Fig. 2), as well as with other galaxy-wide properties such as the ratio of infrared luminosity between stellar birth clouds and the 

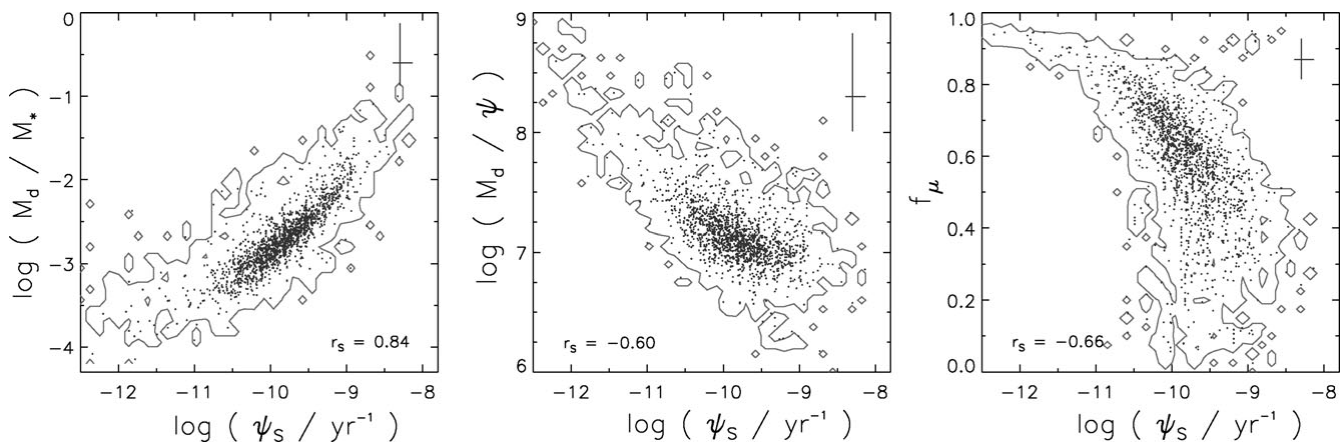

Figure 3. Median-likelihood estimates of three galaxy properties against specific star formation rate, $\psi_{\mathrm{S}}$. Left panel: ratio of dust mass to stellar mass, $M_{\mathrm{d}} / M_{*}$. Middle panel: ratio of dust mass to star formation rate, $M_{\mathrm{d}} / \psi$ (which may be used as a proxy for the dust-to-gas ratio). Right panel: fraction of total infrared luminosity contributed by dust in the ambient ISM, $f_{\mu}$. In each panel, the grey contour shows the distribution of the full matched GALEX-SDSS-2MASS-IRAS sample described in da Cunha et al. (2010), while the points show the distribution of the sub-sample of 1658 galaxies with highest-S/N photometry in all bands. The error bars represent the median confidence ranges in each parameter. The Spearman rank coefficient $r_{S}$ is indicated in each panel.

ambient ISM, the contributions by PAHs and grains in thermal equilibrium to the total infrared emission, and the ratio of dust mass to stellar mass.

\subsection{SDSS-IRAS sample}

We further investigate the relations between star formation activity and dust content using a sample of 3258 star-forming SDSS galaxies with complementary photometric observations by GALEX, 2MASS and IRAS (da Cunha et al. 2009). We find that the specific star formation rate correlates strongly with the dust-to-stellar mass ratio, the ratio of dust mass to star formation rate and the fraction of dust luminosity contributed by the diffuse ISM (Fig. 3). A comparison with recent models of chemical and dust evolution of galaxies suggest that these correlations could arise, at least in part, from an evolutionary sequence (da Cunha et al. 2009).

\section{Acknowledgements}

E. da Cunha acknowledges the IAU for a travel grant and also financial support from the EU ToK grant 39965 and FP7-REGPOT 206469.

\section{References}

Bruzual, G. \& Charlot, S. 2003, MNRAS, 344, 1000

Charlot, S. \& Fall, S. M. 2000, ApJ, 539, 718

da Cunha, E., Charlot, S., \& Elbaz, D. 2008, MNRAS, 388, 1559

da Cunha, E., Eminian, C., Charlot, S., \& Blaizot, J. 2010, MNRAS, in press, arXiv:1001.2309

Dopita, M. A. et al.. 2005, ApJ, 619, 755

Kennicutt Jr., R. C. et al. 2003, PASP, 115, 928

Silva, L., Granato, G. L., Bressan, A., \& Danese, L. 1998, ApJ, 509, 103 